\section{Leukocytoclastic Vasculitis After Influenza Vaccination: An Allergy Assessment}

Barbarroja-Escudero $\mathrm{J}^{1}$, Sánchez-González $\mathrm{MJ}^{1}$, Vélez $\mathrm{D}^{2}$, Aboín $\mathrm{S}^{3}$, Rodríguez-Rodríguez $\mathrm{M}^{1}$, Alvarez-Mon $\mathrm{M}^{1}$

${ }^{I}$ Servicio de Enfermedades del Sistema Inmune-Alergia, Hospital Universitario Príncipe de Asturias, Departamento de Medicina y Especialidades Médicas; Universidad de Alcalá, Alcalá de Henares, Madrid, Spain

${ }^{2}$ Servicio de Anatomía Patológica, Hospital Universitario Príncipe de Asturias; Departamento de Ciencias Morfológicas, Universidad de Alcalá, Alcalá de Henares, Madrid, Spain

${ }^{3}$ Servicio de Dermatología, Hospital Universitario Príncipe de Asturias; Departamento de Medicina y Especialidades Médicas, Universidad de Alcalá, Alcalá de Henares, Madrid, Spain

J Investig Allergol Clin Immunol 2018; Vol. 28(6): 417-418 doi: 10.18176/jiaci.0297

Key words: Influenza vaccine. Intradermal testing. Leukocytoclastic vasculitis. Skin biopsy. TRUE-test.

Palabras clave: Vacuna de la gripe. Prueba intradérmica. Vasculitis leucocitoclástica. Biopsia cutánea. TRUE-test.

Influenza vaccination is indicated for the elderly and patients diagnosed with chronic respiratory disorders, cardiac disease, renal failure, diabetes, and immunosuppression [1]. Although it is a widely accepted and safe practice, influenza vaccine is the most frequent cause of vasculitis in immunizations. Cutaneous vasculitis is the most usual type involved [2]. To our knowledge, this is the first case of leukocytoclastic vasculitis (LCV) following influenza vaccination where an allergy work-up was performed.

The patient was an 86-year-old nonatopic man diagnosed with well-controlled diabetes mellitus, hypertension, and sleep apnea-hypopnea syndrome, with no history of malignancy, autoimmunity, or allergies (chicken meat, egg, latex, or antibiotics). Since 2011, he has experienced episodes of LCV after influenza vaccination (Chiroflu), which was well tolerated on previous occasions. These episodes have become progressively more intense and extensive, occurring more quickly each time and leaving residual hyperpigmentation. In November 2017, 7 days after receiving the last subcutaneous dose on the arm (Chiroflu 2017/2018, Seqirus Vaccines Ltd; A/Michigan/45/2015-H1N1; A/Hong Kong/4081/2014H3N2; B/Brisbane/60/2008), he experienced a new episode of purpuric eruptions on his legs and was referred to the emergency room.

On physical examination, the patient was conscious and alert, with a body temperature of $36.5^{\circ} \mathrm{C}$. Examination of the lungs and abdomen were unremarkable, and no hepatosplenomegaly or peripheral edema was detected. Apart from the skin lesions described, there was also evidence of purpura on both legs that had almost resolved. Laboratory tests only showed mild lymphopenia and macrocytosis, with
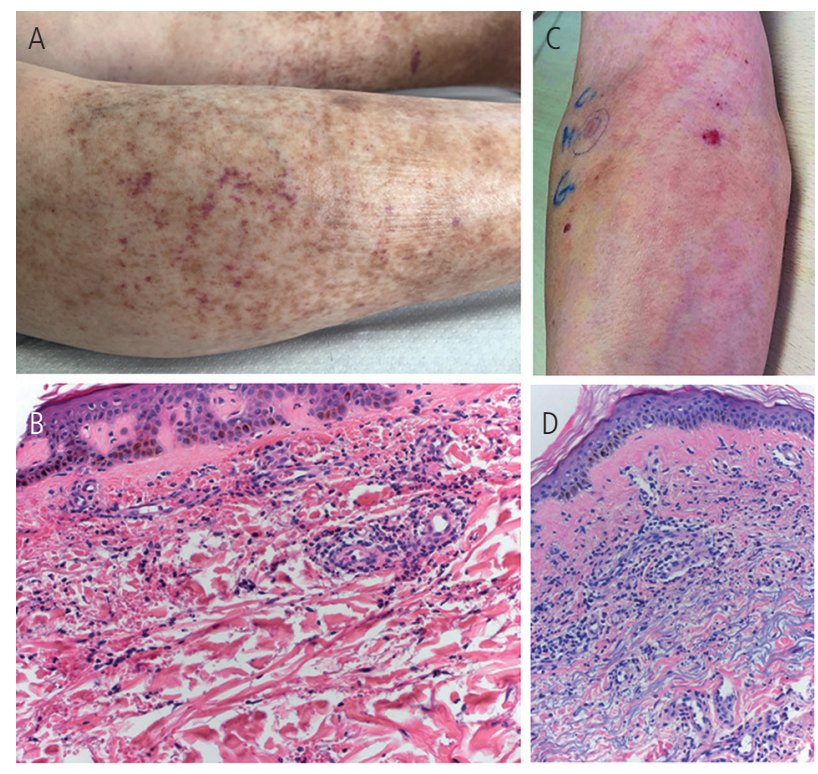

Figure. A, Palpable purpura on the legs. B, Neutrophilic vasculitis of superficial small vessels accompanied by fibrinoid necrosis, conspicuous leukocytoclasia, and extravasation of red cells (hematoxylin-eosin, magnification $\times 20$ ). C, Intradermal test with Chiroflu (1/100) showing a positive result. D, Reaction pattern of small dermal vessels, postcapillary venules, and capillary loops; the blood vessels show fibrinoid necrosis associated with endothelial cell swelling and infiltration by neutrophils and conspicuous nuclear dust (hematoxylin-eosin, magnification $\times 20$ ).

a glucose level of $112 \mathrm{mg} / \mathrm{dL}$. Since neither renal failure nor systemic complications were recorded, therapy was started with prednisone $60 \mathrm{mg} / \mathrm{d}$. A biopsy specimen taken from a new purpuric lesion confirmed a new episode of LCV (Figure, A and B). The treatment was continued for a period of 6 weeks, and the skin lesions resolved within the following few weeks.

The patient was referred to our outpatient clinic in order to evaluate whether the influenza vaccine was responsible for the episodes. In January 2018, general and allergy work-ups were performed. Laboratory tests included complete blood count, hemostasis, erythrocyte sedimentation rate, serum electrolytes, glucose, lipid profile, blood urea nitrogen, creatinine, uric acid, calcium, phosphorus, liver function, thyroid-stimulating hormone, protein electrophoresis, immunoglobulins, rheumatoid factor, C-reactive protein, antinuclear antibodies, antineutrophil cytoplasmic antibody, complement proteins (C3 and C4), hepatitis C and B serology, and urinalysis. They revealed no abnormalities, except for the following parameters (value [normal range]): lymphopenia $\left(850 / \mathrm{mm}^{3}\right.$ [10004800]), erythrocytes (3 $710000 / \mathrm{mm}^{3}$ [4 $\left.\left.500000-5500000\right]\right)$, hemoglobin (12 g/dL [13-18]), hematocrit (35.1\% [37-47]), mean corpuscular volume (94.6 fL [80-90]), mean corpuscular hemoglobin (32.4 pg [25-30]), mean corpuscular hemoglobin concentration (34.2 g/dL [31-34]), platelets (141 000 [150 000$400000])$, complement C4 (<1.72 mg/dL [12-40]), and rheumatoid factor $(504$; normal $<14)$.

Additionally, we performed skin tests with the vaccine (Chiroflu). Skin prick testing yielded a negative result. Subsequently, intradermal testing (IDT, 1/100) and IDT with saline control were carried out, with readings at 15 minutes, 
6 hours, and 24 hours. An ecchymotic papule surrounded by purpuric and marbled exanthema was observed at 24 hours (Figure, C). A patient with a high suspicion of Chiroflu reaction and 2 nonreactive patients who had received the vaccine served as negative controls. A biopsy from the lesion in the positive IDT revealed neutrophilic small vessel vasculitis (Figure, D). Interestingly, there were no episodes of LCV affecting the patient's legs. In February 2018, a new laboratory test was performed with the following results: mean corpuscular volume, $97.9 \mathrm{fL}$; mean corpuscular hemoglobin, $32.5 \mathrm{pg}$; glucose, $141 \mathrm{mg} / \mathrm{dL}$; complement C4, $5.1 \mathrm{mg} / \mathrm{dL}$; and rheumatoid factor, 269. Patch testing (TRUE-Test, Stallergenes Greer Ibérica) and a latex patch test yielded negative results, mainly for formaldehyde, thimerosal, neomycin sulfate, and latex at 48 hours, 72 hours, 96 hours, and 7 days, as previously described $[3,4]$.

$\mathrm{LCV}$, also known as cutaneous small vessel vasculitis or hypersensitivity vasculitis, is the most common vasculitis in adults [5]. Adverse effects of influenza vaccine are recorded in $1 \%-10 \%$ of vaccinated individuals. These are mostly mild and self-limiting (local reactions and constitutional symptoms such as flu-like symptoms) $[1,6]$. Nevertheless, to date, vasculitis after influenza vaccination has been reported in 65 patients, of whom 42 were diagnosed with small vessel vasculitis [7]. The patient we report on here experienced LCV following each annual dose of influenza vaccination. This was accompanied by a decrease in complement $\mathrm{C} 4$ and an increase in rheumatoid factor, both of which remained unchanged for months. Despite the sustained decrease in C4 over time, the clinical and histopathological data did not support urticarial vasculitis as a differential diagnosis.

The mechanisms of onset of LCV remain unclear. In fact, there are cases where an associated eosinophilic infiltration [8,9] or mast cell infiltration [10] has been demonstrated. In the case of neutrophilic vasculitis we present, it seems reasonable to assume direct vessel damage mediated by the vaccine itself (ie, through a virus surface antigen such as hemagglutinin) or indirect damage mediated by memory $\mathrm{T}$ lymphocytes. Moreover, although there was no exacerbation of LCV on the legs after IDT, a reticulated eruption appeared around the positive IDT site (Figure, C), possibly caused by cytotoxic lymphokines. On the other hand, there are no prospective studies on the possible complication (or progress) of LCV secondary to influenza vaccine towards systemic and/ or renal involvement. Therefore, we decided not to administer the influenza vaccine to the patient, despite its benefits in preventing influenza, pneumonia, and, even, death.

In conclusion, to our knowledge, we report the first case of LCV secondary to influenza vaccine in which an allergy work-up was carried out. Given that the use of influenza vaccination is widespread, clinicians should be aware of the possible association with vasculitis, especially in the elderly.

\section{Funding}

This study was partially supported by the Programa de Actividades de I+D between research groups from Comunidad de Madrid en Biomedicina, reference B2017/BMD-3804 "Medicina individualizada traslacional en inflamación y cáncer".

\section{Conflicts of Interest}

The authors declare that they have no conflicts of interest.

\section{References}

1. Ulm S, Hummel M, Emig M, Barreto-Miranda M, Back W, Reiter A, et al. Leukocytoclastic vasculitis and acute renal failure after influenza vaccination in an elderly patient with myelodysplastic syndrome. Onkologie. 2006;29:470-2.

2. Bonetto C, Trotta F, Felicetti P, Alarcón GS, Santuccio C, Bachtiar NS, et al. Vasculitis as an adverse event following immunization - Systematic literature review. Vaccine. 2016;34:6641-51.

3. Johansen JD, Aalto-Korte K, Agner T, Andersen KE, Bircher A, Bruze $M$, et al. European Society of Contact Dermatitis guideline for diagnostic patch testing - recommendations on best practice. Contact Dermatitis. 2015;73:195-221.

4. Wakelin SH, Jenkins RE, Rycroft RJ, McFadden JP, White IR. Patch testing with natural rubber latex. Contact Dermatitis. 1999 Feb;40(2):89-93.

5. D'alò GL, Zorzoli E, Capanna A, Gervasi G, Terracciano E, Zaratti $L$, et al. Frequently asked questions on seven rare adverse events following immunization. J Prev Med Hyg. 2017;58:E13-E26.

6. Yanai-Berar N, Ben-Itzhak O, Gree J, Nakhoul F. Influenza vaccination induced leukocytoclastic vasculitis and pauciimmune crescentic glomerulonephritis. Clin Nephrol. 2002; 58:220-3.

7. Watanabe T. Vasculitis following influenza vaccination: a review of the literature. Curr Rheumatol Rev. 2017;13:18896.

8. Ichiyama S, Funasaka Y, Yamashita H, Tamura H, Inokuchi K, Saeki H. Leukocytoclastic vasculitis with eosinophilic infiltration associated with thalidomide therapy for multiple myeloma: A case report. Allergol Int. 2017;66:497-8.

9. Marzano AV, Tedeschi A, Rossio R, Fanoni D, Cugno M. Prothrombotic state in Churg-Strauss syndrome: a case report. J Investig Allergol Clin Immunol. 2010;20(7):616-9.

10. Inamura $H$, Igarashi $Y$, Kashiwase $Y$, Morioka J, Suzuki $K$, Kurosawa M. Mast cells in cutaneous allergic vasculitis: a case report. Allergol Int. 2006;55:343-5.

Manuscript received May 5, 2018; accepted for publication July 25, 2018.

José Barbarroja-Escudero Servicio de Enfermedades del Sistema Inmune-Alergia, Hospital Universitario Príncipe de Asturias Departamento de Medicina y Especialidades Médicas, Universidad de Alcalá Carretera Madrid-Barcelona, Km 33.600 28805 Alcalá de Henares (Madrid) Spain E-mail: jose.barbarroja@gmail.com; jose.barbarroja@uah.es 